\title{
Efeitos cardiovasculares e respiratórios em cães anestesiados com diferentes concentrações de isoflurano
}

\author{
Cardiovascular and pulmonary effects of different isoflurane \\ concentrations in dogs
}

\author{
Firmino Mársico Filho', Ignacio Alvarez Gomez Segura², Francisco Javier Tendillo², Paulo Roberto Loureiro \\ Nascimento ${ }^{3}$ e Carmen Helena de Carvalho Vasconcellos ${ }^{3}$
}

\begin{abstract}
Resumo
No presente trabalho foram analisados os efeitos sobre os aparelhos cardiovascular e respiratório da anestesia inalatória com isoflurano em 3 (três) concentrações fixas (1. 1,5 e 2 CAM) no cão. Foram utilizados 6 (seis) cães machos, pesando $25 \pm 2,7 \mathrm{~kg}$, com valores hematológicos e bioquímicos dentro da faixa de normalidade. Os parâmetros analisados foram: frequência cardíaca (FC), pressão arterial sistólica (PS), diastólica (PD) e média (PM), débito cardíaco $(\mathrm{DC})$, índice cardíaco (IC), frequência respiratória (FR), volume corrente (VC) e volume minuto (VM). Observou-se que, baixas concentraçōes de isoflurano (1 e 1,5 CAM) produziram um aumento de FC. Com relação à pressão arterial, verificou-se um decréscimo dose-dependente, causado pela diminuição da resistência vascular periférica, o que explicaria a manutenção do DC e IC em baixas concentrações (1 e 1,5 CAM). A 2 CAM de concentração, o aumento da FC não foi capaz de compensar o menor volume de ejeção do coração. Devido a isso, observou-se queda do DC e IC. No sistema respiratório, encontrou-se diminuição da FR, VC e VM, dose-dependente.
\end{abstract}

Palavras-chave: isoflurano, efeitos cardiopulmonares, anestesia, cão

\section{Introdução}

A utilização dos barbitúricos como agentes anestésicos únicos, supôs em um momento, um avanço extraordinário, devido principalmente às vantagens que proporcionava, como baixo custo, facilidade de administração e o fato de, teoricamente, poder-se prescindir do anestesista. Desta forma, se converteu na técnica de eleição durante certo tempo.
Esta prática, com o passar do tempo, demonstrou inconvinientes, como a falta de relaxamento muscular, o débil poder analgésico e a forma de administração, que dificultava a manutenção do plano anestésico e a possibilidade de ocorrência de sobredosificão. Além disso, por serem metabolizados por via hepática e renal, depositando-se em tecidos adiposos, os barbitúricos podem levar a despertar muito prolongado em pacientes com afecções hepato-renais ou simplesmente obesos.

Em 1957, o descobrimento do halotano, agente inalatório halogenado, mudou radicalmente o conceito e a metodologia anestesiológica. Apesar de seus inconvenientes e efeitos indesejáveis, como depressão cardiovascular, sensibilização do miocárdio às catecolaminas, hepato e nefrotoxicidade, além da hipertermia maligna relatada em algumas espécies, o halotano apresenta múltiplas vantagens, que o tornam amplamente utilizado até hoje.

A partir deste primeiro halogenado, foram sintetizados outros agentes, como o metoxiflurano e o enflurano, até que, em 1965, Ross Terrel sintetizou o isoflurano. Estudos prévios deste fármaco informaram erroneamente sua capacidade oncogênica (Corbett, 1976) levando ao seu abandono até 1981, quando foi autorizada sua comercialização e demonstradas suas vantagens em relação aos outros halogenados, principalmente quanto aos seus efeitos cardiovasculares e sua metabolização.

Estes dois últimos pontos tornam a utilização do isoflurano na Medicina Veterinária cada vez mais intensa, principalmente nos animais que apresentam risco anestésico por serem portadores de processos patológicos derivados do aparetho cardiovascular e/ou hepato-renal e alterações neurológicas.

\footnotetext{
1 Prof. Doutor Adjunto de Técnica Cirúrgica e Anestesiologia, da Fac. de Veterinária/UFF. Rua Vital Brazil Filho 64, Niterói - RJ. CEP. 24230-340

2 Profs. Doutores da Universidade Complutense de Madrid

${ }_{3}^{3}$ Médicos-Veterinários, alunos de Pós-Graduação em Cirurgia Veterinária, UFF
} 
O presente trabalho tem como objetivo, avaliar a influência de três diferentes concentrações de isoflurano sobre a função cárdio-respiratória, em cães.

\section{Material e Métodos}

Foram utilizados 6 cães machos, hígidos, com pesos variando entre 22,3 e 27,7kg. Observou-se jejum alimentar de 12 horas e privação hídrica de 4 horas.

Padronizou-se a Concentração Alveolar Mínima (CAM) de isoflurano em 1,5\%, de acordo com Eger (1981). Previamente à indução anestésica, administrou-se oxigênio através de máscara de Hall durante 3 minutos, após os quais foi iniciada a administração de isoflurano, com fluxo de 6 litros de $\mathrm{O}_{2}$ por minuto. A concentração de isoflurano foi aumentada de $0,5 \%$ a cada 20 segundos até alcançar $5 \%$. Após a perda dos reflexos, realizou-se a intubação endotraqueal, conectando-se a sonda a um circuito circular com absorção de oxigênio ${ }^{4}$. Posteriormente, a concentração do anestésico foi reduzida a 2 CAM e, em seguida, cateterizaram-se as vias arterial e venosa (ramos da artéria e veia femural) sob condições de antissepia rigorosas. Infiltrou-se lidocaína a 2\% ao redor da incisão cirúrgica com a finalidade de se evitar uma possível estimulação dolorosa que levasse a alterações nos valores cardiopulmonares analisados.

A tomada dos valores foi realizada, sequencialmente, nas concentrações de 1, 1,5 e 2 CAM do anestésico. A comprovação da concentração de isoflurano foi feita através de um espectrofotômetro de infravermelho ${ }^{5}$, que aferiu a quantidade de isoflurano ao final da expiração. Os valores foram mensurados aos 5, 10, 15 e 20 minutos para cada concentração do anestésico.

As pressões arterial sistólica (PS) e diastólica (PD), foram mensuradas através de um transdutor de pressão conectado ao cateter na artéria femural ${ }^{6}$ e a frequência cardíaca (FC), por eletrocardiograma na derivação II. O débito cardíaco (DC) foi determinado pelo método de termodiluição ${ }^{7}$ com cateter de Swan-Ganz. Antes de cada tomada de valores, realizou-se um lavado em ambos os cateteres com soro fisiológico heparinizado $(5 \mathrm{Ul} / \mathrm{ml})$. A frequência respiratória (FR) e o volume minuto (VM) foram aferidos por espirômetro de Wright. Os valores de pressão média (PM), índice cardíaco (IC) e volume corrente (VC) foram calculados, respectivamente, através das fórmulas: (PS+2PD/3), (DC/peso) e (FR/MM).

Ao término da cada estudo, foram realizadas as ligaduras das colaterais da veia e da artéria femural com fio de seda 3-0 e as suturas do subcutâneo (fio catgut 3-0) e pele (seda 3-0).

Os resultados obtidos foram submetidos a tratamento estatístico ${ }^{8}$ que constou de análise de variância, correlação linear de Pearson e teste de Duncan, para $\mathrm{P}<0,05$.

\footnotetext{
${ }^{4}$ Boyle absorver Mark III, British Oxygen Co.Ltd.

${ }^{5}$ A. M. Bickford Inc.

${ }^{6}$ Monitor de ECG e pressão sanguínea: Patient Monitor General Eletric

${ }^{7}$ Computador de Débito Cardíaco, Edwards

${ }^{8}$ SAEG versão 3.0, UFV
}

\section{Resultados}

Durante a fase de indução com máscara, praticamente todos os animais apresentaram breve período de excitação. Não foram detectadas arritmias durante a fase de indução.

Os resultados relacionados aos parâmetros cardiovasculares e respiratórios, estão representados pelo Quadro 1 e Figs. 1 a 5. As colunas assinaladas com asterisco apre-

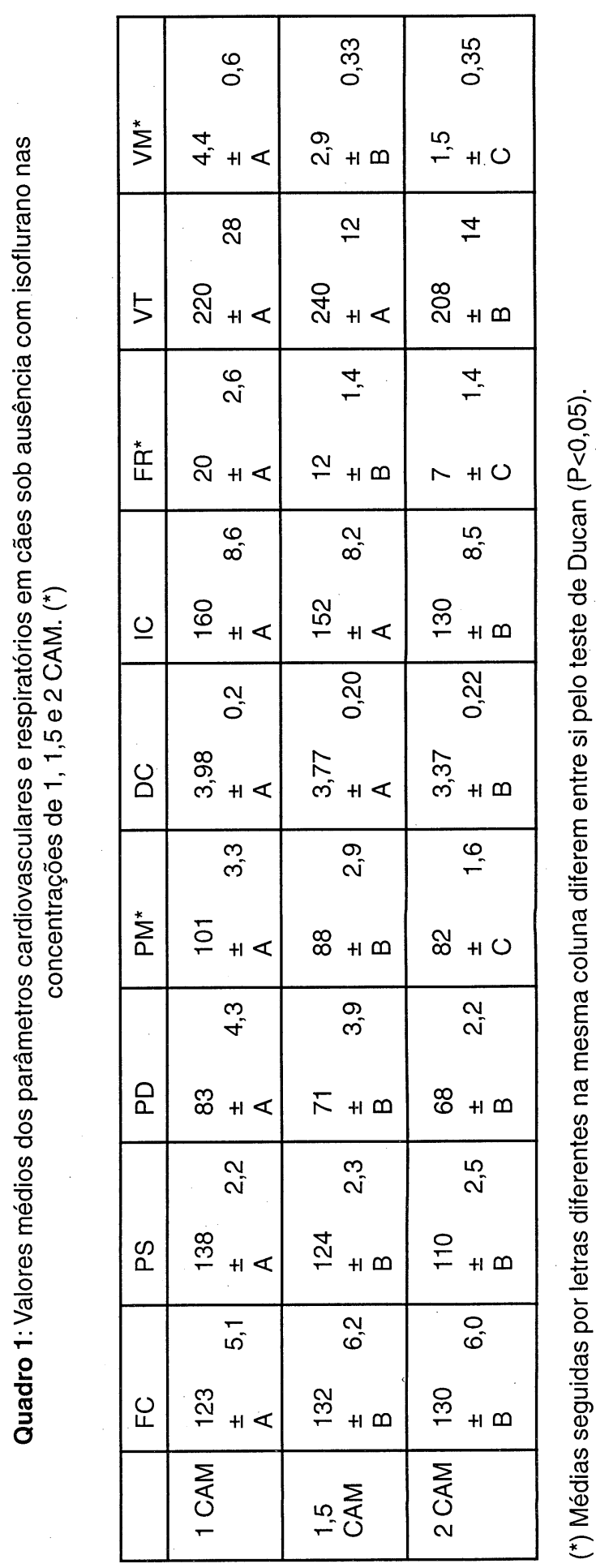


sentam variação significativa entre os valores encontrados em diferentes concentrações de isoflurano.

Os valores de pressão média, frequência respiratória e volume minuto, apresentaram variações significativas entre as 3 concentrações de isoflurano. Foram encontradas ainda, diferenças entre alguns valores de frequência cardíaca,

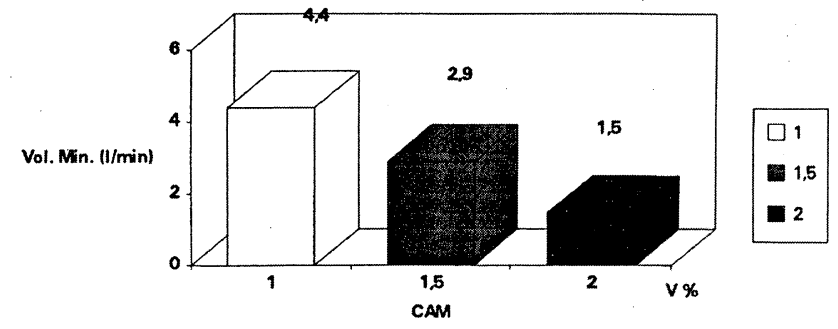

Fig. 1: Valores médios de volume minuto ( $/ / \mathrm{min})$ em cães anestesiados com 3 concentrações de isoflurano.

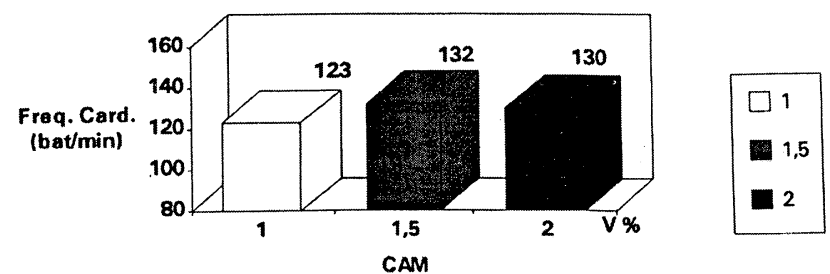

Fig. 2: Valores médios de frequência cardíaca (bat/min) em cães anestesiados com 3 concentrações de isoflurano.

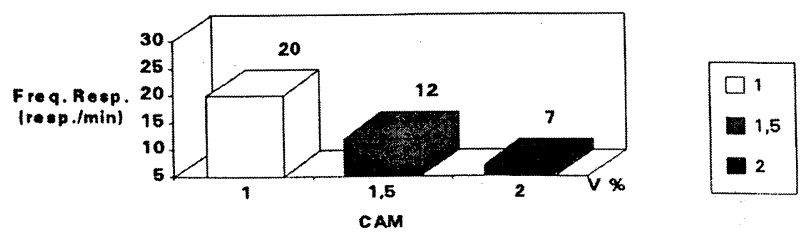

Fig. 3: Valores médios de frequência respiratória (resp/min) em cães anestesiados com 3 concentrações de isoflurano.

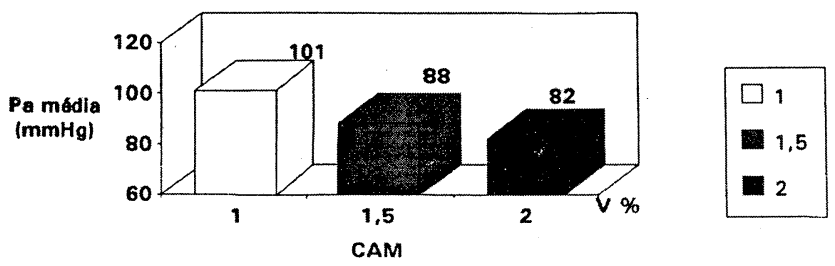

Fig. 4: Valores médios de pressão arterial média $(\mathrm{mm} / \mathrm{Hg})$ em cães anestesiados com 3 concentrações de isoflurano

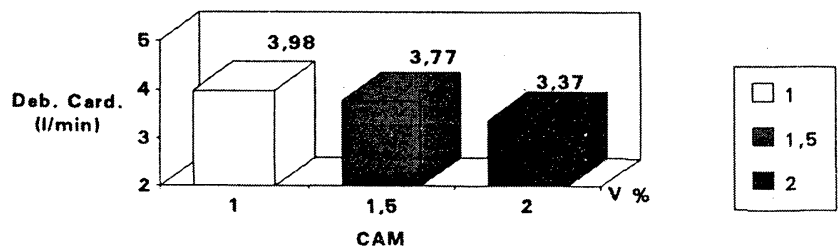

Fig. 5: Valores médios de débito cardíaco ( $/ / \mathrm{min})$ em cães anestesiados com 3 concentrações de isoflurano. pressão sistólica, pressão diastólica, débito cardíaco, índice cardíaco e volume corrente.

\section{Discussão}

Os anestésicos halogenados utilizados comumente, produzem marcada depressão cardiovascular (Klide, 1976; Steffey et al., 1977; Jones \& Snowdon, 1986) e respiratória (Fourcade et al., 1974; Steffey \& Howland, 1977; Jones \& Snowdon, 1986), dose-dependente. Os resultados encontrados neste trabälho confirmam estas observações, sendo mais acentuado o efeito sobre a função respiratória.

O isoflurano apresenta características que o tornam mais indicado que outros agentes halogenados, como o halotano e o enflurano. As principais vantagens se baseiam em indução e recuperação mais rápida e menor grau de metabolismo, devido à sua absorção e eliminação pulomonares. Atualmente existe controvérsias sobre os efeitos cardiovasculares e respiratórios destes agentes e, se o isoflurano representa clara vantagem sobre os outros agentes halogenados. Aparentemente, o isoflurano produz menor efeito depressor cardiovascular que o halotano em doses anestésicas habituais (1 e 1,5 CAM) (Steffey et al., 1987), ao contrário, existem evidências que indicam maior depressão respiratória, dose-dependente, com o isoflurano (Higgs \& Carli, 1983).

Segundo alguns autores (Klide, 1976; Steffey \& Howland, 1977; Jones \& Snowdon, 1986), baixas concentrações de isoflurano produzem aumento da frequência cardíaca em cães. Tal fato foi observado no presente trabalho, nas concentrações de 1,5 e 2 CAM, quando comparados com 1 CAM, sendo causado, provavelmente por beta-estimulação (Perry et al., 1974) ou estímulo dos barorreceptores (Skousted \& Sapthauichaikul, 1977). Este aumento da frequência cardíaca associado à diminuição da pressão arterial por redução da resistência vascular periférica (Klide, 1976; Jones \& Snowdon, 1986), explicaria a ausência de modificações no débito cardíaco, observadas com concentraçōes baixas do anestésico e sua manutenção, até que as concentrações atinjam 2 CAM $(P<0,05)$. Nestas concentrações, o aumento da frequência cardíaca não é capaz de compensar o menor volume de ejeção do coração (Klide, 1976; Steffey \& Howland, 1977).

O sistema respiratório também sofreu depressão. Baixas concentrações de isoflurano (1,5 CAM) levaram a decréscimo do volume minuto; prodúzido por queda da frequência respiratória (Steffey \& Howland, 1977). Concentrações superiores (2 CAM) ocasionaram ainda diminuição do volume corrente (Steffey \& Howland, 1977), o que reduziu ainda mais o volume minuto.

\section{Abstract \\ Cardiovascular and pulmonary effects of different isoflurane concentrations in dogs}

Cardiovascular and pulmonary effexts of isoflurane anesthesia at 1, 1,5 and 2 minimum alveolar concentration (MAC) were studies in 6 male dogs, healty, weighting $25 \pm$ $2,7 \mathrm{~kg}$. Heart rate $(H R)$, systolic arterial pressure (SAP), 
dyastolic arterial pressure rate (RR), tidal volume (TV) and minute ventilation (MV) were measured. Low isoflurane concentrations (1 and 1,5 MAC) increased the heart rate (HR). Arterial blood pressure decreased as the MAC increased, explaining the maintenance of the $\mathrm{CO}$ and the $\mathrm{Cl}$ at low concentrations of isoflurane (1 and 1,5 MAC). At 2 MAC the increase of the HR is not enough to compensate the low heart stroke volume, so a drop of $\mathrm{CO}$ an $\mathrm{Cl}$ was observed. A dose-dependent decrease of the RR, TV and MV was also observed.

Key words: isoflurane, cardiopulmonary effects, anesthesia, dog

\section{Referências Bibliográficas}

CORBET, T. H. Cancer and congenital anomalies associated with anesthetics. Ann.NY Acad. Sci., v. 271, p. 58-66, 1976.

EGER II, E. I. Isoflurane: a review. Anesthesiology, v.55, p. 559-576, 1981. HIGGS, B. D., CARLI, F. An analysis of ventilatory response to carbon dioxide with halotane and isoflurane anesthesia. Anesthesiology, v. 59, n. 3, p. $487,1983$.
JONES, R. S., SNOWDON, S. L. Experimental investigation of the cardiovascular and respiratory effects of increasing concentrations of isoflurane in the dog. Res. Vet. SCl., v. 40, p. 89-93, 1986

KLIDE, A. M. Cardiopulmonary effects of enflurane and isoflurane in the dogs. Am. J. Vet. Res., v. 37, p. 127-131, 1976.

PERRY, L. B., VAN DYKE, R. A., THEYE, R. A. Sympathoadrenal hemodynamic effects of isoflurane, halotane and cyclopropane in dogs. Anesthesiology, v. 40, p. 465-470, 1974.

SKOUSTED, P., SAPTHAUICHAIKUL, S. The effects of isoflurane on arterial pressure, pulse rate, autonomic nervous activity and barostatic. Can. Anaesth. Soc. J., v. 24, p. 304-314, 1977.

STEFFEY, E. P., HOWLAND JR, D. Isoflurane in dog and cat. Am. J. Vet. Res., v. 38, p. 1833-1836, 1977.

STEFFEY, E. P., HOWLAND JR, D., GIRI, S., EGER II, E. I. Enflurane, halotane and isoflurane potency in horses. Am. J. Vet. Res., v.38, p.1037-1039, 1977.

STEFFEY, E. P., DUNLOP, C. I., FARVER, T. B., NOLINER, M. J., SCHULTZ, L. J. Cardiovascular and respiratory measurements in awake and isofluraneanesthetized horses. Am. J. Vet. Res., v. 48, p. 7-12, 1987. 